ECCOMAS

Proceedia
COMPDYN 2021

$8^{\text {th }}$ ECCOMAS Thematic Conference on Computational Methods in Structural Dynamics and Earthquake Engineering M. Papadrakakis, M. Fragiadakis (eds.)

\title{
ON THE DEVELOPMENT OF A NOVEL APPROACH FOR SIMULATING ELASTIC BEAMS IN DUALSPHYSICS WITH THE USE OF THE PROJECT CHRONO LIBRARY
}

Salvatore Capasso ${ }^{1 *}$, Bonaventura Tagliafierro ${ }^{1}$, Iván Martínez-Estévez ${ }^{2}$, José M. Domínguez $^{2}$, Joe El Rahi ${ }^{3}$, Vasiliki Stratigaki ${ }^{3}$, Alejandro J.C. Crespo ${ }^{2}$, Rosario Montuori ${ }^{1}$, Peter Troch $^{3}$, Moncho Gómez-Gesteira ${ }^{2}$, Giacomo Viccione ${ }^{1}$

${ }^{1}$ University of Salerno

Via Giovanni Paolo II, 132, 84084 Fisciano, Italy

capassosalva7@gmail.com,btagliafierro@unisa.it,gviccione@unisa.it,r.montuori@unisa.it

${ }^{2}$ Universidade de Vigo

Campus As Lagoas s/n, 32004, Ourense, Spain

ivan.martinez.estevez@uvigo.es, jmdominguez@uvigo.es, alexbexe@uvigo.es,mggesteira@uvigo.es

${ }^{3}$ Ghent University

Department of Civil Engineering, 9000 Belgium

Joe.ElRahi@ugent.be, Vasiliki.stratigaki@ugent.be, Peter.Troch@UGent.be

\begin{abstract}
This work presents a novel numerical framework for dynamic analyses of structure systems within the meshless approach Smoothed Particle Hydrodynamics (SPH) method. The Lagrangian solver DualSPHysics presents several advantages over the widely used Eulerian solvers, dealing with nonlinearities and multiphase phenomena with reasonable numerical stability and reliability. The proposed procedure exploits the mechanical features provided by the Project Chrono library to simulate elastic beams. The modelling procedure is of interest for studying complex soil-, solid-, fluid-structure interactions, involving a system that includes all of the aforementioned phases in a unitary context. The analytical formulation to pass information over to the SPH solver for generating a sub-assembly of rigid stubs and elastic hinges, that will mimic the behavior of a Euler-Bernoulli flexible beam, is presented. The approach is validated against theoretical Euler-Bernoulli solutions: the agreement between the theoretical solutions for the behavior of the flexible beams and the presented model is very good and increases when the number of elements that make up the beam, $N$, increases. In addition, the behavior of the flexible beam thus created in the SPH environment is validated considering a sensitivity analysis based on several parameters, such as the model resolution (initial interparticle distance) and the number of elements.
\end{abstract}

Keywords: SPH, Euler-Bernoulli, Linear Elasticity, FSI, CFD, DualSPHysics, Project Chrono. 


\section{INTRODUCTION}

The Meshfree Particle Methods (MPMs) present several advantages over mesh-based methods, like the natural ability to solve multi-mechanics problems: the Lagrangian nature of this family of numerical methods allows to compute, with relative ease, multi-mechanics problems involving complex interfaces and moving boundaries. In particular, the Smoothed Particle Hydrodynamics (SPH) method, is, by now, well established in the Computational Fluid Dynamics (CFD) discipline, and its effectiveness has been proven in several works, especially when dealing with free-surfaces flows and large deformations [1].

The DualSPHysics code has been applied to several multiphysics phenomena, for example in coastal engineering simulations: to compute forces exerted by large waves on the urban furniture of a realistic promenade [2], to study the run-up on a real armour block coastal breakwater [3, 4] and to simulate large waves generated by land-slide events [5]. Other successful applications of DualSPHysics are performed in the field of renewable energies, for example the simulation of Wave Energy Converters (WECs) in several contexts: from the study of the moorings and floatings dynamics [6, 7, 8, 9], to the efficiency [10] and survivability [11] analysis when combined with closed loops [12, 13], PTO systems [4, 14] or non linear mechanical constraints [15]. These works present first attempts of reproducing the effects of power take-off systems through simplified, although reliable, approaches. Specific validation of Fluid Structure Interactions (FSI) in DualSPHysics are provided by [16]. However, an option to model flexible objects is not yet available.

In this work, the potentiality of an SPH-based solver, DualSPHysics [17], augmented with the Project Chrono library [18], developed as a general purpose simulation framework for multibody problems, are utilized to simulate the flexural behavior of one-dimensional beams. Other approaches to this issue, with the SPH formulation, can be found in several works (for example, [19, 20, 21, 22]). Rigid bodies and mutual constraints allow developing an alternative framework for the dynamics analysis of structural systems, able to reproduce extremely high deformations of flexible elements, under complex and time-variant load conditions, or impacts - useful for simulating the response of engineered structures under the menaces of hazardous natural events. The aforementioned numerical architecture, in fact, provides a robust and reliable framework for studying fluid-solid interaction, handling arbitrarily defined fluid-structurestructure coupled systems, consenting to specify mutual and/or absolute constraints, such as joints and sliders. SPH is a suitable method for modelling water-related natural hazards due to its characteristics $[23,24]$.

The proposed beam model relies on a lumped elasticity formulation, in which the only deformation allowed is represented by the relative rotation between consecutive trunks. A similar representation can be found in [25], while a valid application of this formulation to structure collapse is presented in [26]. To improve this work, following [25] and [26], the interactions between the rigid bodies, modelled as mutual constraints, can be characterized by elastic, then plastic behavior, up to a threshold value of stress that determines the collapse. For the peculiarities of the Lagrangian-based method here presented, the latter situation is reproducible with relative ease, encouraging further investigations. In the SPH-based framework, the dynamics of the trunks, and so of the beam as a whole, depends on the Newton's equations for rigid bodies, computed taking into account the mutual constraints and the interaction with external forces. A numerical algorithm developed from classical static theories is here proposed (i.e. EulerBernoulli (EB) beam theory), and implemented in DualSPHysics to simulate linear elasticity, using the features provided by the coupling with Project Chrono. In the following, after the 
presentation of the SPH formulation implemented in the DualSPHysics code, and its coupling with Project Chrono, the theoretical approach is formulated and validated against EB solutions. Then, the proposed approach is reproduced in DualSPHysics by combining hinges and rigid bodies and their response in investigated.

\section{SPH FORMULATION}

MPMs in general refers to the class of meshfree methods that employ a set of finite number of discrete particles to represent the state of a system and to record its movement. Each particle can either be directly associated with one discrete physical object, or be generated to represent a part of the continuum problem domain. For CFD problems, each particle possesses a set of field variables such as mass, momentum, energy, positions etc., and other variables (vorticity, etc.) related to the specific problem. The advantages of the MPMs methods over conventional grid-based numerical methods can be roughly summarized as follows:

- the problem domain is discretized with particles without a fixed connectivity, so treatment of large deformation is relatively easier;

- discretization of complex geometry is simpler as only an initial discretization is required;

- it is easy to obtain the features of the entire physical system through tracing the motion of the particles, therefore, identifying free surfaces, moving interfaces and deformable boundaries, is no longer a tough task.

Among the MPMs, the Smoothed Particle Hydrodynamics (SPH) method is employed in the present work.

\subsection{Principles of the SPH method}

The strategy in SPH is to discretize the physical domain (fluid and/or solid objects) into a set of particles, where the physical quantities (position, velocity, density and pressure) are obtained as an interpolation of the corresponding quantities of the surrounding particles. The contribution of those particles is weighted using a kernel function, with an area of influence that is defined using a characteristic smoothing length. This discretization process is divided into two key steps [27].

The first step is the integral representation or the so-called kernel approximation of field functions, consisting in the integration of a multiplication of an arbitrary function and a smoothing kernel function. The integral representation of a generic spatial function $f(\mathbf{r})$ within an integral volume $\Omega$, is given by:

$$
<f(\mathbf{r})>=\int_{\Omega} f\left(\mathbf{r}^{\prime}\right) W\left(\mathbf{r}-\mathbf{r}^{\prime}, h\right) d \mathbf{r}^{\prime}
$$

where $W$ is the so-called smoothing kernel function or kernel. In the smoothing function, $h$ is the smoothing length defining the influence area of $W$ (Figure 1).

The second step is the particle approximation. The integral representation is approximated by summing up the values of the nearest neighbor particles, which yields the particle approximation of the function at a discrete point (particle). The position $\mathbf{r}_{b}$ is defined as the position of a particle having a fixed mass $m_{b}$ and a finite volume $V_{b}$, related by

$$
V_{b}=\frac{m_{b}}{\rho_{b}}
$$


where $\rho_{b}$ is the density of particle $b=1, \ldots, N_{p}$ in which $N_{p}$ is the number of particles within the support domain of particle $a$. The integral representation can be written in a discrete form for a particle $a, b$ being part of its support domain:

$$
<f\left(\mathbf{r}_{a}\right)>=\sum_{b=1}^{N_{p}} \frac{m_{b}}{\rho_{b}} f\left(\mathbf{r}_{b}\right) W_{a b}
$$

where:

$$
W_{a b}=W\left(\mathbf{r}_{a}-\mathbf{r}_{b}, h\right)
$$

The kernel function, hence, plays a fundamental role in the SPH method. In DualSPHysics the Wendland [28] quintic kernel function is utilized:

$$
W(r, h)=\alpha_{D, n}\left\{\begin{array}{rr}
\left(1-\frac{q}{2}\right)^{4}(1+2 q) & 0 \leq q \leq 2 \\
0 & 2<q
\end{array}\right.
$$

where:

$$
q=\frac{r}{h}=\frac{\left|\mathbf{r}-\mathbf{r}^{\prime}\right|}{h}
$$

and $\alpha_{D, n}$ is a constant depending on the dimension of the problem.

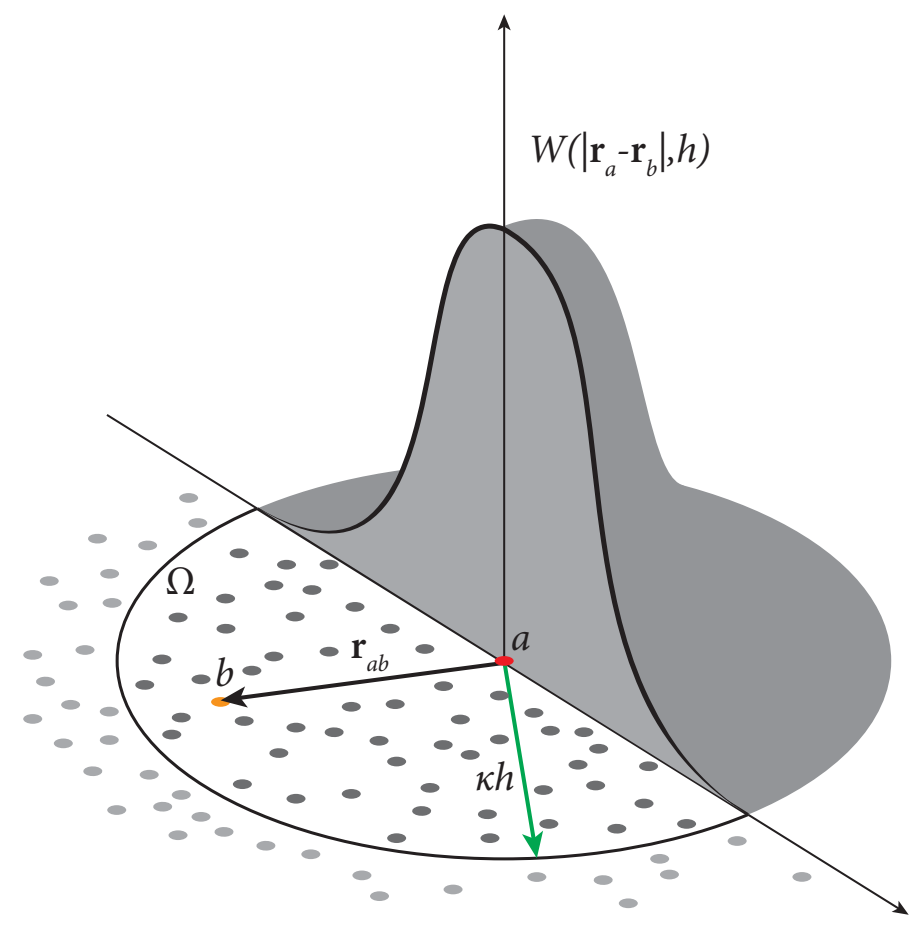

Figure 1: Smoothing kernel function.

\subsection{Governing equations for rigid bodies}

Rigid bodies are sub-sets of SPH particles, the variables of which are integrated in time with the Newton's equations for rigid body dynamics, in the domain frame reference. 
The equations of motion are:

$$
\begin{aligned}
M \frac{d \mathbf{V}}{d t} & =\mathbf{F}_{\Omega} \\
\mathbf{J} \frac{d \boldsymbol{\Omega}}{d t} & =\mathbf{F}_{\Omega}
\end{aligned}
$$

where $\mathbf{F}_{\Omega}$ is the total moment with respect to the center of mass, and they assume the following aspect in particle approximation:

$$
\begin{array}{r}
M_{I} \frac{d \mathbf{V}_{I}}{d t}=\sum_{k \in I} m_{k} \frac{d \mathbf{u}_{k}}{d t} \\
\mathbf{J}_{I} \frac{d \boldsymbol{\Omega}_{I}}{d t}=\sum_{k \in I} m_{k}\left(\mathbf{r}_{k}-\mathbf{R}_{I}\right) \times \frac{d \mathbf{u}_{k}}{d t}
\end{array}
$$

where the body I posses a mass $M_{I}$, velocity $\mathbf{V}_{I}$, inertia tensor $\mathbf{J}_{I}$, angular velocity $\Omega_{I}$ and center of gravity $\mathbf{R}_{I}$. The vectorial quantities are computed at every time step (discretizing the temporal derivative $\frac{d}{d t}$ ). The force by unit mass applied to the particle $k$ belonging to $I$ is expressed as $m_{k} \frac{d \mathbf{u}_{k}}{d t}$, encompassing body forces (gravity) and external loads resultants. The net force on each boundary particle is computed according to the sum of the contributions of all surrounding fluid particles according to the designated kernel function and smoothing length. It is clear that every particle within the body has a velocity given by:

$$
\mathbf{u}_{k}=\mathbf{V}+\Omega_{I} \times\left(\mathbf{r}_{k}-\mathbf{R}_{I}\right)
$$

These equations are integrated in times over a staggered interval, in DualSPHysics, using a computationally simple Verlet based scheme that guarantees a second order spatial accuracy. For more details about rigid bodies in DualSPHysics, [29] is suggested.

\subsection{Coupling scheme}

Mechanisms involving contacts and impacts between parts can be modeled in terms of multibody systems with unilateral constraints. Considering the success of SPH for fluid descriptions and non-smooth multi-body solvers for mechanical systems, attempting to couple both under a generalized framework should provide new simulation possibilities, by leveraging the strengths of both methods. Project Chrono is able to simulate a wide range of physical problems, but at the moment, DualSPHysics uses the rigid body, constraints and collision detection parts of the library. This allows to use a set of bodies described in meshes, define restrictions applied to the bodies (from the implemented list of joints, hinges and springs) and compute interactions between bodies, in a similar fashion of the DEM implementation, but in a more stable manner.

The implementation strategy is to couple both models with a message passing interface. Once quantities from the rigid bodies are computed by DualSPHysics (Equation (8)), the time step, along with the linear and angular accelerations from each body, are sent to the Project Chrono module [16]. For that time step, Chrono returns the linear and angular velocities, as well as centres of mass position, computed by integrating the fluid contributions with the dynamic or kinematic restrictions of the system, including collisions.

\section{LUMPED ELASTICITY FORMULATION}

The developed approach is based on the discretization of a one-dimensional element (Figure 2) defined under the assumptions of the EB theory, which are here briefly recalled. Being $x$ the abscissa that identifies the beam axis: 
- the longitudinal axis of the unloaded, undeformed beam, is rectilinear;

- small shifts;

- inalterability of the cross section, i.e. always planar;

- the generic cross section $\Sigma(x)$ is always orthogonal to the axis, also in the deformed configuration;

- isotropic elastic material;

- $x$ dimension predominant with respect to $y, z$.

As a consequence of these assumptions, considering the behavior of the beam to be only flexural, the function which uniquely describes its deformation is the vertical displacement function $v=v(z)$, namely the elastic line equation (Figure 2). Furthermore, every cross section is characterized by infinitesimal rigid displacements, expressible as a summation of a translation $(v)$ and a rotation $(\vartheta)$ around its centre of mass $G$.

(a)

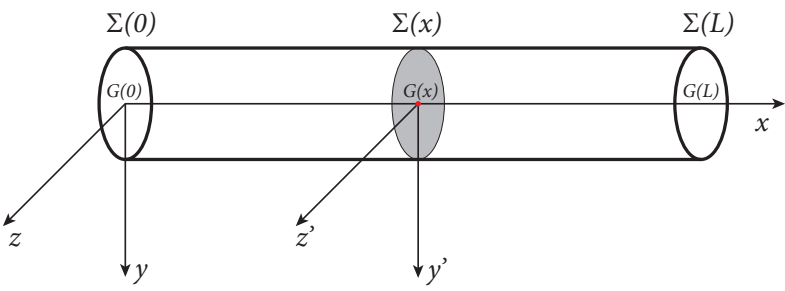

(b) $\quad \Sigma(x)$

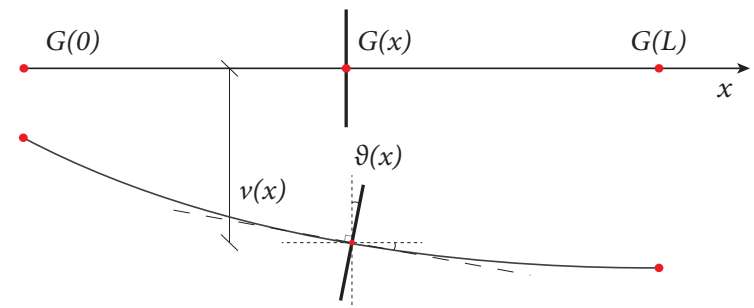

Figure 2: Representation of a beam with rectilinear axis (a), and relative kinematic parameters (b).

Following these considerations, it is possible to reproduce the displacement function with the finite element representation, in which the elements assume a physical dimension, and thus the latter are governed by the rigid body kinematics. In fact, a generic derivable function, in this case $v=v(x)$, can be replaced with a series of local defined low-order functions $\psi_{i}$, which interpolate between the $v\left(x_{i}\right)=v_{i}$ values:

$$
v(z) \approx \sum_{i} \psi_{i} v_{i}
$$

In case $\psi_{i}$ is a first order polynomial, the approximation is linear, which can be materially represented by rigid elements, from now on trunks. The numerical properties of the interpolation polynomials are physically the positions and the lengths of the trunks, whose ending cross section are equipped with rotational hinges. The hinge positions, related to a generic trunk, identified respectively as $i$ and $i+1$, are expressible with a single rotational parameter, namely $\vartheta_{i}$ (Figure 3 ). The displacement of the point $i+1$ is known, once the the angle $\vartheta_{i}$ (rotation in $i$ ) is note; from the Equation (9) we have:

$$
\mathbf{u}_{i+1}=\Omega_{i} \times \mathbf{r}_{i, i+1}
$$

which becomes in a two-dimensional EB framework, being $r_{i, i+1}=r_{i+1}-r_{i} \doteq \Delta x$, and assuming $\Omega=\left\{0,0, \vartheta_{i}\right\}$ :

$$
v_{i+1}=\sin \vartheta_{i} \Delta x
$$


The proposed numerical model is elaborated for a cantilever, but its approximations can be extended to any kind of Boundary Conditions (BC). This particular statical scheme was chosen because several FSI problems present models akin to this one, and also various validation processes are based on cantilevers (see $[30,31]$ ). The cantilever, hence, is discretized as a set of rigid trunks and rotational hinges [25]. The hinge is characterized by a proper value of rotational stiffness $K_{\vartheta, i}$, constant with the length of the trunk $\Delta x$, that is deduced from the geometrical e mechanical properties of the section.

(a)

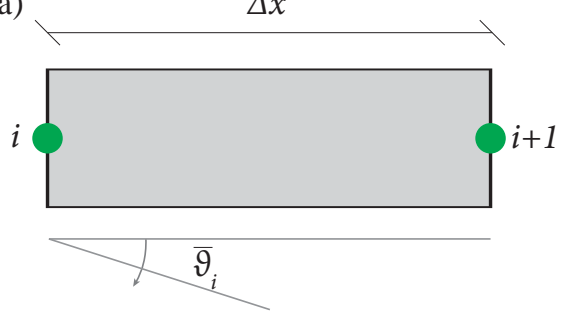

(b)

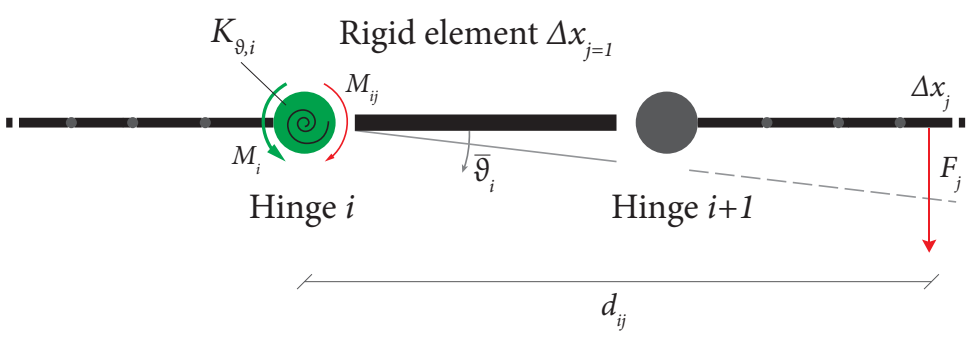

Figure 3: Vertical displacement of the end of a rigid trunk (a), and discretization scheme (b).

\subsection{Numerical discretization}

Let us consider the cantilever section in the plane $\{x, y\}$, of length $L_{s}$ and thickness $h_{b}$, the beam axis being axis of symmetry and coincident with $x$. Let $A_{s}=h_{s} b_{s}$ be the area of the cross section, assumed rectangular and constant, $b_{s}$ being the base, aligned with $x, I_{s}$ the moment of inertia, $E_{s}$ and $\rho_{s}$ rispectively the Young's modulus and density of the material. The beam is arbitrarily divided in $N$ (identified with the subscript $j$ ) trunks $\Delta x$, linked with $N$ (identified with the subscript $i$ ) hinges (Figure 5). In case of constant spatial discretization, we can define:

$$
\Delta x=\frac{L_{s}}{N}
$$

and the generic abscissa is $x(i) \doteq x_{i}$. Within this framework, following the assumption that the behavior of the beam is only flexural, as visible in the elastic line equation:

$$
\frac{d^{2} v(x)}{d x^{2}}=-\frac{M(x)}{E_{s} I_{s}}
$$

the moment is the only components of tension to be dealt with. The moment $M_{i j}$ is defined as the moment created by the $j-t h$ resultant with respect to the $i-t h$ hinge:

$$
M_{i j}=d_{i j} F_{j}
$$

where $d_{i j}$ is the lever arm of the force $F_{j}$, applied in the center of mass $G_{j}$ of the trunk, with $G_{j}>x_{i}$. The total moment referred to the $i-t h$ hinge is:

$$
M_{i} \doteq \sum_{k=j}^{N} M_{i k}=\sum_{k=j}^{N} d_{i k} F_{k}
$$

The local rotation $\bar{\vartheta}_{i}$ of the $i-t h$ hinge is proportional to the total moment calculated in the section $x_{i}$, by the rotational stiffness, following the theory of linear elasticity:

$$
\bar{\vartheta}_{i}=\frac{M_{i}}{K_{\vartheta, i}}
$$


This value, which characterizes the flexural behavior of each trunk, can be computed once the external load is known. To secure a correct numerical result for a cantilever scheme, the rotational stiffness of the bearing (first section) has to be doubled with respect to the generic stiffness value, since the first trunk can be considered semi-fixed. Thanks to rigid body kinematics is possible, then, to obtain the complete displacement function of the beam; let

$$
\vartheta_{i}=\sum_{k=1}^{i} \bar{\vartheta}_{k}
$$

be the total rotation of the $i-t h$ hinge;

$$
\bar{v}_{i+1}=\Delta x_{j=i} \sin \vartheta_{i}
$$

is, hence, the local vertical displacement of the section $x_{i+1}$, and

$$
v_{i}=\sum_{k=1}^{i} \bar{v}_{k}
$$

is the total vertical displacement.

This numerical approach, being based on an equilibrium (Equation (17)), can be naturally extended in the dynamic framework of DualSPHysics and Project Chrono, once defined the governing parameters to be transmitted: number and dimensions of the trunks and the stiffness related to each hinge. To complete the implementation of the one-dimensional elastic element in DualSPHysics, the resolution parameter $d p$ has to be defined, namely the initial inter-particle distance, as function of the beam thickness $h_{s}$ :

$$
d p \doteq \frac{h_{s}}{p p\left(h_{s}\right)}
$$

where $p p\left(h_{s}\right)$ is the number of particles per straight section.

\section{Validation}

The use of the SPH formulation to discretize one-dimensional flexible elements has two main, and consequential, objectives:

1. demonstrate that a fixed set of particles is able to reproduce, properly, the behavior of a rigid element, subject to active (force $\frac{d \mathbf{u}_{k}}{d t}$ applied to the $k-t h$ particle, computed with Equation (8) by DualSPHysics) and reactive (forces exerted by the mutual and absolute constraints, computed by the Project Chrono module) forces, and that several rigid elements and links can be combined to mimic one-dimensional flexible beams;

2. proved the first statement, the potentialities of the SPH method can be exploited: due to the use of rigid body dynamics, high order elasticity formulations are not required to compute extremely high deformations; the natural ability to reproduce free-surface flows, impacts, complex interfaces, can be used, then, to investigate fluid elastic-structure interactions (for example [20, 32, 33]).

The validation is based on a static comparison between the analytical and numerical displacement function of a cantilever: being the DualSPHysics framework dynamic, the results of 
a static case can be generalized with ease to a wide field of dynamic conditions. The potentialities of this approach in the SPH framework are, then, shown in several works, which represent various possible physical contests, like interaction with free-surfaces flows or dynamics impacts with fluid mass (reproducing a dambreak flow impacting a flexible obstacle [31, 33]).

\subsection{Cantilever test case: static validation}

Here the graphics of the statical deflection of the beam, compared against the EB solution, are exposed. The elastic line equation for a cantilever gives:

$$
v_{a n}(x)=\frac{q_{s} L_{s}^{2} x^{2}}{4 E_{s} I_{s}}-\frac{q_{s} L_{s} x^{3}}{6 E_{s} I_{s}}+\frac{q_{s} x^{4}}{24 E_{s} I_{s}}
$$

where $q_{s}=q_{s}(x)$ is the vertical load (only gravitational load, in this case).

The sensitivity analysis on the model is conducted with variable number of trunks $(N)$ and with different values of resolution. The displacement function in the DualSPHysics dynamic framework is obtained by adding a linear spring element at the free end of the cantilever, as schematized in Figure 4. The circles in Figure 4(b) represent the hinges, that mutually link the trunks, identified with the color bar and a number $m k$.

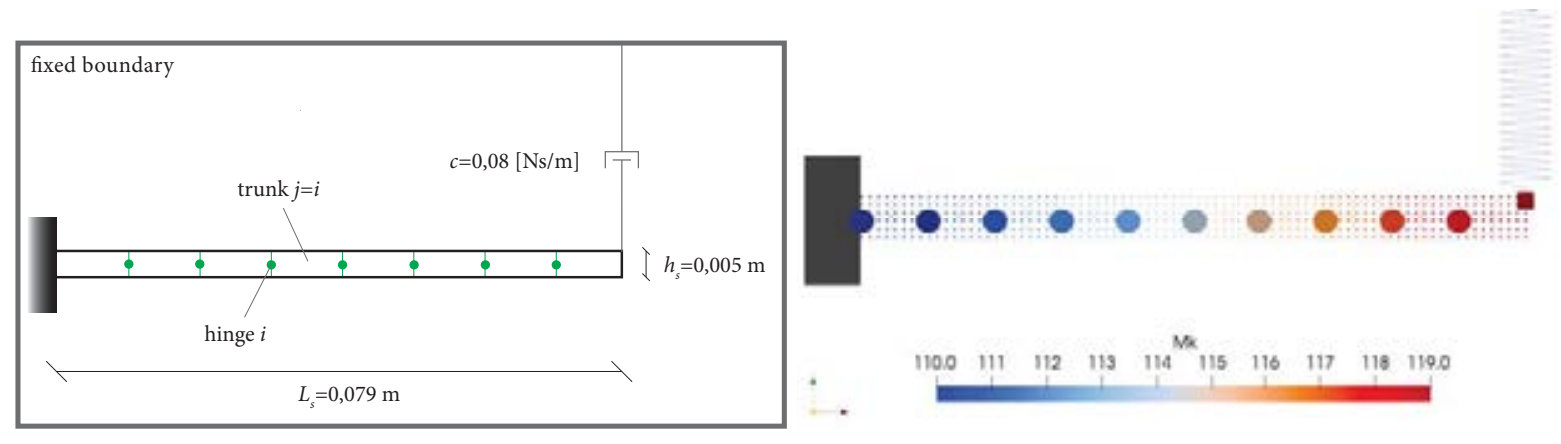

(a)

(b)

Figure 4: Simulation set-up (a) and relative SPH model (b).

The ulterior constraint works uniquely as a liner damper, being the stiffness value set to zero, thus affecting only the velocity of the oscillation and yielding to the steady state for the system. In the two-dimensional $\{O, x, z\}$ space of our simulations, the force exerted by the additional constraint is:

$$
F_{l s}=c u_{v, z}
$$

where $c$ is the damping, considering the cantilever axis parallel to $x$ axis.

In the Table 4.1, the model information necessary for the DualSPHysics simulations is recalled, with clear significance of the nomenclature. The simulation shows the rising agreement, in the SPH framework, depending on the number of trunks. The agreement is good, and the model can be considered effective with the first value of resolution,

$$
d p=\frac{0.005}{5}=0.001[\mathrm{~m}]
$$

the displacement function being properly fitted (Figure 5(a)), despite a small deviation around the centerline, regardless of the number of trunks. This is highlighted in the zoom (Figure 6(a)). By doubling the resolution, instead, the numerical solution is perfectly reproduced (Figure 5(b)). 


\begin{tabular}{cc}
\hline \multicolumn{2}{c}{ Dimensions } \\
\hline$L_{s}[\mathrm{~m}]$ & 0.079 \\
$b_{s}[\mathrm{~m}]$ & 0.005 \\
$h_{s}[\mathrm{~m}]$ & 0.005 \\
\hline Physical characteristics & \\
\hline$\rho_{s}\left[\mathrm{~kg} / \mathrm{m}^{3}\right]$ & 1100 \\
$E_{s}\left[\mathrm{~N} / \mathrm{m}^{2}\right]$ & $1.2 \mathrm{E}+7$ \\
$g\left[\mathrm{~m} / \mathrm{s}^{2}\right]$ & 9.81 \\
$c[\mathrm{Ns} / \mathrm{m}]$ & 0.08 \\
\hline
\end{tabular}

Table 1: Dimension and physical characteristics of the cantilever.
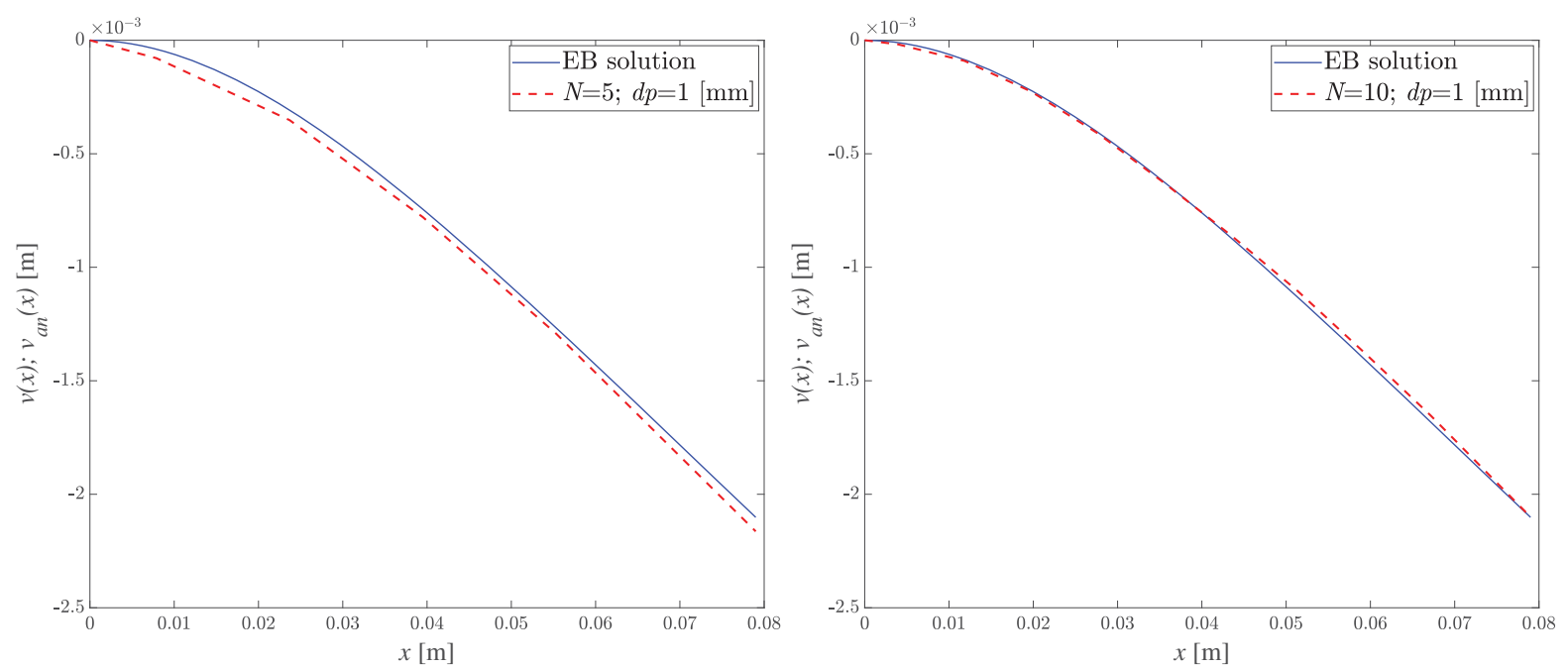

(a)
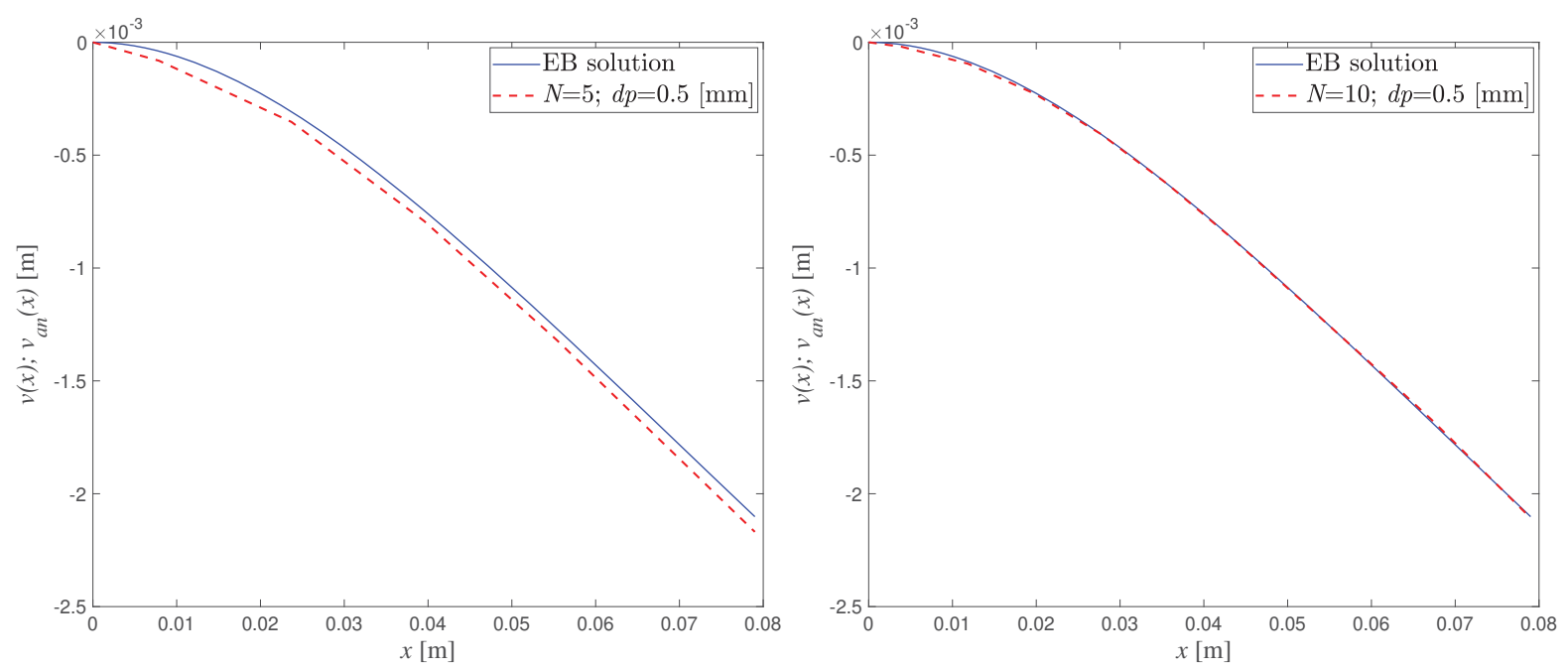

(b)

Figure 5: Comparison between the DualSPHysics results and the analytic solution: first value of resolution $(d p=$ $1[\mathrm{~mm}])(\mathrm{a})$, and second value of resolution $(d p=0.5[\mathrm{~mm}])(\mathrm{b})$.

It is safe to say, after this test, that the software is able to simulate the static displacement function of an EB cantilever, and the model converges with the analytical solution as the number 
of trunks increases, as visible in Figure 6. With a higher number of particles the precision is very high (Figure 6(b)), but more costly in terms of computation, since the resolution value is unique for all the simulated domain. The particle analysis on the beam model shows that beyond a certain value of particles per cross section, the agreement remains unchanged. These two values, the number of trunks and the number of particles, influence the computational effort: the former by increasing the number of interactions to be computed by the Project Chrono module, the latter by increasing the number of discrete entities where the field variables have to be calculated by DualSPHysics, with more neighbors within each support domain. With $N=10$, as seen in Figure 5, the model is already effective in simulating one-dimensional beams; for what concerns the resolution value, an adequate value of $p p\left(h_{s}\right)$ is enough to guarantee the numerical stability. It means that, in complex simulation involving fluid domains, the resolution needed for the beam constitutes the lower limit, which could be increased if required by other relevant conditions, just as the number of trunks, which can be raised if there are particular situations or a very high precision is required.

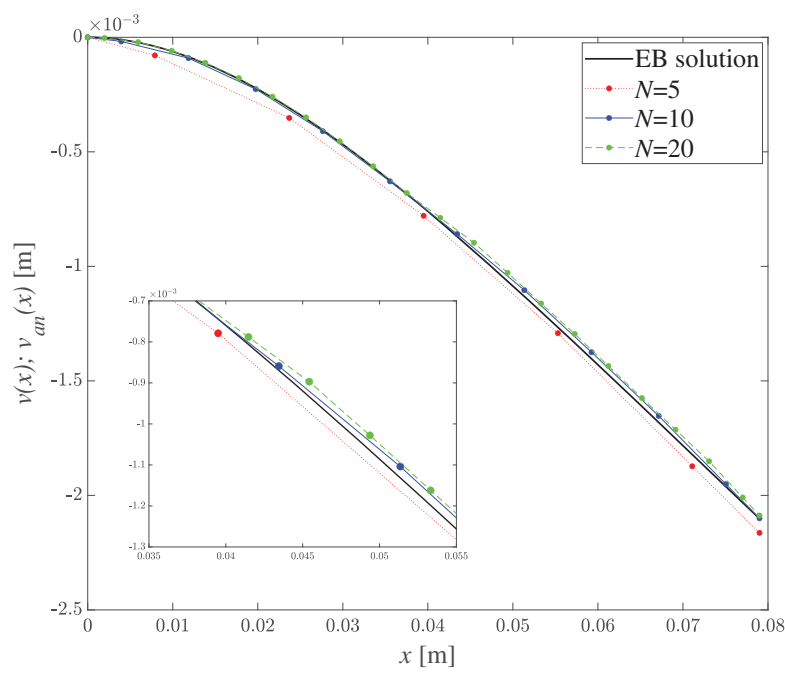

(a)

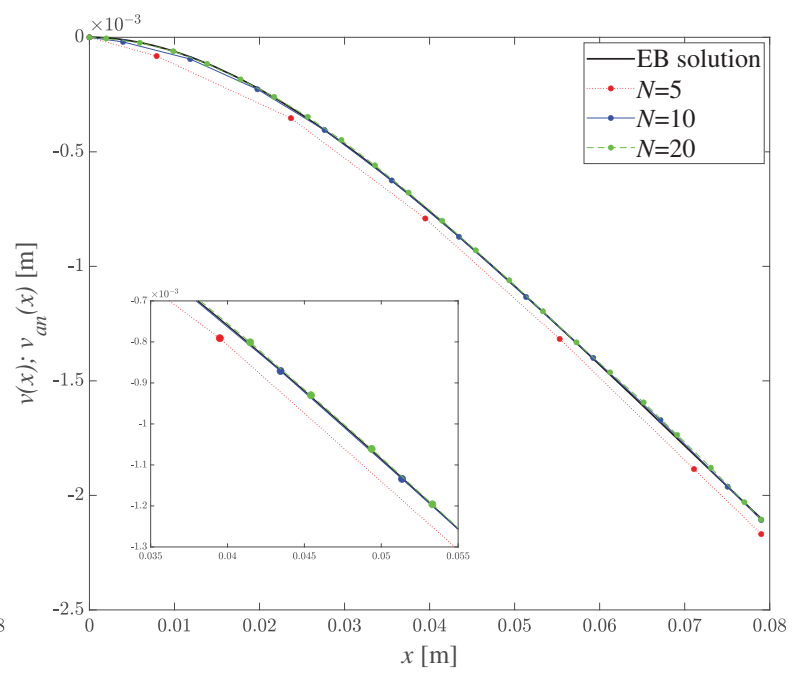

(b)

Figure 6: Convergence analysis of DualSPHysics results: first value of resolution $(d p=1[\mathrm{~mm}])$ (a), and second value of resolution $(d p=0.5[\mathrm{~mm}])(\mathrm{b})$.

\section{CONCLUSIONS AND FUTURE WORK}

The results show very good agreement between the analytic solutions and the presented model, highlighting its applicability to elastic cantilevers, or comparable static schemes, interacting with fluid or, in general, subject to dynamic impacts, time-variant load conditions, causing extreme deformations and high order effects. The numerous advantages of the SPH formulation, hence, allow thorough investigation of structure models, based on the proposed lumped elasticity discretization.

The solid framework of DualSPHysics and Project Chrono, moreover, offers the possibility to simulate complex multiphysics problems, making it suit to real-world engineering problems. Possible application fields are:

- flow-structure interactions of coastal vegetation;

- swinging of wind turbine columns subject to waves or seismic action; 
- modelling of vibrations of one-dimensional structures interacting with Newtonian or nonNewtonian fluids; and

- structure collapse.

In conclusion, the simplicity, the effectiveness and the wide possibilities of implementation, in several fields, make this model a valuable starting point to investigate fluid elastic-structure interactions, or, more widely, elastic structure dynamics.

\section{REFERENCES}

[1] D. Violeau and B.D. Rogers. Smoothed particle hydrodynamics (sph) for free-surface flows: past, present and future. Journal of Hydraulic Research, 54(1):1-26, 2016. doi: 10.1080/00221686.2015.1119209.

[2] A. Barreiro, A.J.C. Crespo, J.M. Domínguez, and M. Gómez-Gesteira. Smoothed particle hydrodynamics for coastal engineering problems. Computers \& Structures, 120:96-106, 2013. ISSN 0045-7949. doi: https://doi.org/10.1016/j.compstruc.2013. 02.010. URL https://wwW.sciencedirect.com/science/article/pii/ s0045794913000588.

[3] C. Altomare, A.J.C. Crespo, B.D. Rogers, J.M. Dominguez, X. Gironella, and M. GómezGesteira. Numerical modelling of armour block sea breakwater with smoothed particle hydrodynamics. Computers \& Structures, 130:34-45, 2014. ISSN 0045-7949. doi: https: //doi.org/10.1016/j.compstruc.2013.10.011. URL https : / / www . sciencedirect. com/science/article/pii/s0045794913002824.

[4] Z. Zang, Q. Zhang, Y. Qi, and X. Fu. Hydrodynamic responses and efficiency analyses of a heaving-buoy wave energy converter with pto damping in regular and irregular waves. Renewable Energy, 116:527 - 542, 2018. ISSN 0960-1481. doi: https://doi.org/10.1016/ j.renene.2017.09.057.

[5] Renato Vacondio, Benedict Rogers, P.K. Stansby, Paolo Mignosa, and Jon Feldman. Variable resolution for sph: A dynamic particle coalescing and splitting scheme. Computer Methods in Applied Mechanics and Engineering, 256:132-148, 04 2013. doi: 10.1016/j.cma.2012.12.014.

[6] Minghao Wu, Vicky Stratigaki, Tim Verbrugghe, Peter Troch, Corrado Altomare, Alejandro Crespo, Dogan Kisacik, Lorenzo Cappietti, José Dominguez, Matthew Hall, Moncho Gómez-Gesteira, Peter Stanstby, Ricarod Birjukovs Canelas, and Rui Ferreira. Experimental study of motion and mooring behavior of a floating oscillating water column wave energy converter. 2018.

[7] Minghao Wu, Vasiliki Stratigaki, Peter Troch, Corrado Altomare, Tim Verbrugghe, Alejandro Crespo, Lorenzo Cappietti, Matthew Hall, and Moncho Gómez-Gesteira. Experimental study of a moored floating oscillating water column wave-energy converter and of a moored cubic box. Energies, 12(10), 2019. ISSN 1996-1073. doi: 10.3390/en12101834.

[8] J.M. Domínguez, A.J.C. Crespo, M. Hall, C. Altomare, M. Wu, V. Stratigaki, P. Troch, L. Cappietti, and M. Gómez-Gesteira. Sph simulation of floating structures with moorings. Coastal Engineering, 153:103560, 2019. ISSN 0378-3839. doi: https://doi.org/10.1016/j. coastaleng.2019.103560. 
[9] Alejandro Crespo, Matthew Hall, J.M. Domínguez, Corrado Altomare, Minghao Wu, Tim Verbrugghe, Vasiliki Stratigaki, Peter Troch, and Moncho Gómez-Gesteira. Floating moored oscillating water column with meshless sph method. pages 11-53, 062018. doi: 10.1115/OMAE2018-77313.

[10] N. Quartier, P. Ropero-Giralda, J.M. Domínguez, V. Stratigaki, and P. Troch. Influence of the drag force on the average absorbed power of heaving wave energy converters using smoothed particle hydrodynamics. Water (Switzerland), 13(3), 2021. doi: $10.3390 /$ w 13030384 .

[11] D. Kisacik, V. Stratigaki, M. Wu, L. Cappietti, I. Simonetti, P. Troch, A. Crespo, C. Altomare, J.M. Domínguez, M. Hall, M. Gómez-Gesteira, R. Canelas, and P.K. Stansby. Efficiency and survivability of a floating oscillating water column wave energy converter moored to the seabed: An overview of the esflowc marinet2 database. Water, 12:992, 04 2020. doi: 10.3390/w12040992.

[12] Pablo Ropero-Giralda, Alejandro J.C. Crespo, Bonaventura Tagliafierro, Corrado Altomare, José M. Domínguez, Moncho Gómez-Gesteira, and Giacomo Viccione. Efficiency and survivability analysis of a point-absorber wave energy converter using dualsphysics. Renewable Energy, 162:1763 - 1776, 2020. ISSN 0960-1481. doi: https: //doi.org/10.1016/j.renene.2020.10.012. URL http: / / www. sciencedirect.com/ science/article/pii/s0960148120315780.

[13] Pablo Ropero-Giralda, Alejandro J. C. Crespo, Ryan G. Coe, Bonaventura Tagliafierro, José M. Domínguez, Giorgio Bacelli, and Moncho Gómez-Gesteira. Modelling a heaving point-absorber with a closed-loop control system using the dualsphysics code. Energies, 14(3), 2021. ISSN 1996-1073. doi: 10.3390/en14030760. URL https : / / www . mdpi . $\mathrm{com} / 1996-1073 / 14 / 3 / 760$.

[14] Bonaventura Tagliafierro, Rosario Montuori, Ioannis Vayas, Pablo Ropero-Giralda, Alejandro Crespo, José Domìnguez, Corrado Altomare, Giacomo Viccione, and Moncho Gòmez-Gesteira. A new open source solver for modelling fluid-structure interaction: case study of a point-absorber wave energy converter with a power take-off unit. In Proceedings of the $11^{\text {th }}$ International Conference on Structural Dynamics, Athens, Greece, 11 2020. doi: 10.47964/1120.9052.21578.

[15] M. Brito, R.B. Canelas, O. García-Feal, J.M. Domínguez, A.J.C. Crespo, R.M.L. Ferreira, M.G. Neves, and L. Teixeira. A numerical tool for modelling oscillating wave surge converter with nonlinear mechanical constraints. Renewable Energy, 146:2024 - 2043, 2020. ISSN 0960-1481. doi: https://doi.org/10.1016/j.renene.2019.08.034.

[16] R.B. Canelas, M. Brito, O.G. Feal, J.M. Domínguez, and A.J.C. Crespo. Extending dualsphysics with a differential variational inequality: modeling fluid-mechanism interaction. Applied Ocean Research, 76:88 - 97, 2018. ISSN 0141-1187. doi: https: //doi.org/10.1016/j.apor.2018.04.015.

[17] J.M. Domínguez, G. Fourtakas, Corrado Altomare, Ricardo Canelas, Angelo Tafuni, Orlando García Feal, Iván Martínez-Estévez, A. Mokos, Renato Vacondio, Alejandro Crespo, Benedict Rogers, P.K. Stansby, and M. Gómez-Gesteira. Dualsphysics: from fluid 
dynamics to multiphysics problems. Computational Particle Mechanics, 03 2021. doi: 10.1007/s40571-021-00404-2.

[18] Alessandro Tasora, Radu Serban, Hammad Mazhar, Arman Pazouki, Daniel Melanz, Jonathan Fleischmann, Michael Taylor, Hiroyuki Sugiyama, and Dan Negrut. Chrono: An open source multi-physics dynamics engine. pages 19-49, 06 2016. ISBN 978-3-31940360-1. doi: 10.1007/978-3-319-40361-8_2.

[19] Carla Antoci, Mario Gallati, and Stefano Sibilla. Numerical simulation of fluid-structure interaction by sph. Computers Structures, 85(11):879-890, 2007. ISSN 0045-7949. doi: https://doi.org/10.1016/j.compstruc.2007.01. 002. URL https://www.sciencedirect.com/science/article/pii/ S0045794907000132. Fourth MIT Conference on Computational Fluid and Solid Mechanics.

[20] G. Fourey, C. Hermange, D. Le Touzé, and G. Oger. An efficient fsi coupling strategy between smoothed particle hydrodynamics and finite element methods. Computer Physics Communications, 217:66-81, 2017. ISSN 0010-4655. doi: https://doi.org/10.1016/j.cpc. 2017.04.005. URL https://www.sciencedirect.com/science/article/ pii/s0010465517301194.

[21] Abbas Khayyer, Hitoshi Gotoh, Hosein Falahaty, and Yuma Shimizu. An enhanced isphsph coupled method for simulation of incompressible fluid-elastic structure interactions. Computer Physics Communications, 232, 06 2018. doi: 10.1016/j.cpc.2018.05.012.

[22] Abbas Khayyer, Yuma Shimizu, Hitoshi Gotoh, and Shunsuke Hattori. Multi-resolution isph-sph for accurate and efficient simulation of hydroelastic fluid-structure interactions in ocean engineering. Ocean Engineering, 226:108652, 2021. ISSN 0029-8018. doi: https: //doi.org/10.1016/j.oceaneng.2021.108652. URL https: //www. sciencedirect. com/science/article/pii/s0029801821000871.

[23] Sauro Manenti, Dong Wang, J.M. Domínguez, S. Li, Andrea Amicarelli, and Raffaele Albano. Sph modeling of water-related natural hazards. Water, 11:1875, 09 2019. doi: 10.3390/w11091875.

[24] A. Amicarelli, S. Manenti, R. Albano, G. Agate, M. Paggi, L. Longoni, D. Mirauda, L. Ziane, G. Viccione, S. Todeschini, A. Sole, L.M. Baldini, D. Brambilla, M. Papini, M.C. Khellaf, B. Tagliafierro, L. Sarno, and G. Pirovano. Sphera v.9.0.0: A computational fluid dynamics research code, based on the smoothed particle hydrodynamics mesh-less method. Computer Physics Communications, 250:107157, 2020. ISSN 0010-4655. doi: https://doi.org/10.1016/j.cpc.2020.107157.

[25] Piseth Heng, Anas Alhasawi, Jean-Marc Battini, and Mohammed Hjiaj. Co-rotating rigid beam with generalized plastic hinges for the nonlinear dynamic analysis of planar framed structures subjected to impact loading. Finite Elements in Analysis and Design, 157:38-49, 2019. ISSN 0168-874X. doi: https://doi.org/10.1016/j.finel.2018. 11.003. URL https://www.sciencedirect.com/science/article/pii/ S0168874X17305966. 
[26] Dan Dubina, Ioan Marginean, and Florea Dinu. Impact modelling for progressive collapse assessment of selective rack systems. Thin-Walled Structures, 143:106201, 2019. ISSN 0263-8231. doi: https://doi.org/10.1016/j.tws.2019.106201. URL https : / / www . sciencedirect.com/science/article/pii/s0263823118316021.

[27] G.R. Liu and M.B. Liu. Smoothed Particle Hydrodynamics: A Meshfree Particle Method. 01 2003. doi: 10.1142/5340.

[28] H. Wendland. Piecewise polynomial, positive definite and compactly supported radial basis functions of minimal degree. Advances in Computational Mathematics, 4(1):389396, December 1995. doi: 10.1007/BF02123482. URL http://sro. sussex.ac . uk/id/eprint/24323/.

[29] Ricardo B. Canelas, Jose M. Domínguez, Alejandro J.C. Crespo, Moncho GómezGesteira, and Rui M.L. Ferreira. A smooth particle hydrodynamics discretization for the modelling of free surface flows and rigid body dynamics. International Journal for $\mathrm{Nu}$ merical Methods in Fluids, 78(9):581-593, 2015. doi: https://doi.org/10.1002/fld.4031.

[30] Stefan Turek and Jaroslav Hron. Proposal for Numerical Benchmarking of Fluid-Structure Interaction Between an Elastic Object and Laminar Incompressible Flow, volume 53, pages 371-385. 06 2007. ISBN 978-3-540-34595-4. doi: 10.1007/ 3-540-34596-5_15.

[31] Kangping Liao, Changhong Hu, and Makoto Sueyoshi. Free surface flow impacting on an elastic structure: Experiment versus numerical simulation. Applied Ocean Research, 50:192-208, 2015. ISSN 0141-1187. doi: https://doi.org/10.1016/j.apor.2015. 02.002. URL https://www.sciencedirect.com/science/article/pii/ S0141118715000231.

[32] Peng-Nan Sun, David Le Touzé, Guillaume Oger, and A-Man Zhang. An accurate fsi-sph modeling of challenging fluid-structure interaction problems in two and three dimensions. Ocean Engineering, 221:108552, 2021. ISSN 0029-8018. doi: https://doi.org/10.1016/ j.oceaneng.2020.108552. URL https: / /www.sciencedirect.com/science/ article/pii/s0029801820314608.

[33] P.N. Sun, D. Le Touzé, and A.-M. Zhang. Study of a complex fluid-structure dam-breaking benchmark problem using a multi-phase sph method with apr. Engineering Analysis with Boundary Elements, 104:240-258, 2019. ISSN 0955-7997. doi: https://doi.org/ 10.1016/j.enganabound.2019.03.033. URL https: / / www. sciencedirect.com/ science/article/pii/s0955799719300943. 